\title{
L'emergenza del fenomeno urbano nell'Europa continentale della seconda età del ferro: Bibracte e gli oppida celtici
}

L'émergence du phénomène urbain dans l'Europe continentale au deuxième âge du fer: Bibracte et les oppida celtiques

The Emergence of the Urban Phenomenon in Continental Europe during the Second Iron Age: Bibracte and the Celtic Oppida

\section{Andrea Fochesato}

\section{(2) OpenEdition}

\section{Journals}

Edizione digitale

URL: https://journals.openedition.org/gaia/1136

DOI: 10.4000/gaia. 1136

ISSN: 2275-4776

Editore

UGA Éditions/Université Grenoble Alpes

Edizione cartacea

ISBN: 978-2-37747-199-7

ISSN: $1287-3349$

Notizia bibliografica digitale

Andrea Fochesato, «L'emergenza del fenomeno urbano nell'Europa continentale della seconda età del ferro: Bibracte e gli oppida celtici», Gaia [Online], 22-23 | 2020, online dal 30 juin 2020, consultato il 09 décembre 2021. URL: http://journals.openedition.org/gaia/1136 ; DOI: https://doi.org/10.4000/ gaia.1136

Questo documento è stato generato automaticamente il 9 décembre 2021.

Gaia. Revue interdisciplinaire sur la Grèce archaïque 


\title{
L'emergenza del fenomeno urbano nell'Europa continentale della seconda età del ferro: Bibracte e gli oppida celtici
}

\author{
L'émergence du phénomène urbain dans l'Europe continentale au deuxième âge \\ du fer: Bibracte et les oppida celtiques \\ The Emergence of the Urban Phenomenon in Continental Europe during the \\ Second Iron Age: Bibracte and the Celtic Oppida
}

Andrea Fochesato

\section{La nascita dell'idea di una città celtica: la «civilisation des oppida»}

L'utilizzo del sostantivo oppida per definire dei siti fortificati a carattere urbano caratteristici del popolamento della tarda età del ferro a nord delle Alpi (periodo tardo latèniano, fine del II e I secolo a.C., fig. 1) trova la sua origine nei Commentarii cesariani, sola testimonianza diretta pervenutaci sul mondo celtico transalpino del I secolo a.C. In un celebre passo del primo libro ${ }^{1}$, il proconsole distingue tre forme di insediamento - gli aedificia, i vici e gli oppida (tradotte generalmente come fattorie, villaggi aperti e città, per lo più fortificate $\left.{ }^{2}\right)$ - in un contesto di occupazione gerarchizzata del territorio delle civitates galliche che pone gli oppida in posizione di vertice. Dalla lettura del testo cesariano si evince come questi ultimi siano innanzitutto dei siti di rifugio a funzione militare, ma anche poli di stoccaggio e scambio economico in cui è possibile trovare rifornimenti per l'esercito ${ }^{3}$, oltre che centri politici nei quali si tengono assemblee, riunioni del senato e dove si decidono le sorti della guerra ${ }^{4}$. 
Fig. 1. - Carta della diffusione degli oppida e delle agglomerazioni non fortificate di III e II secolo a.C., con indicazione dei siti citati nel testo.

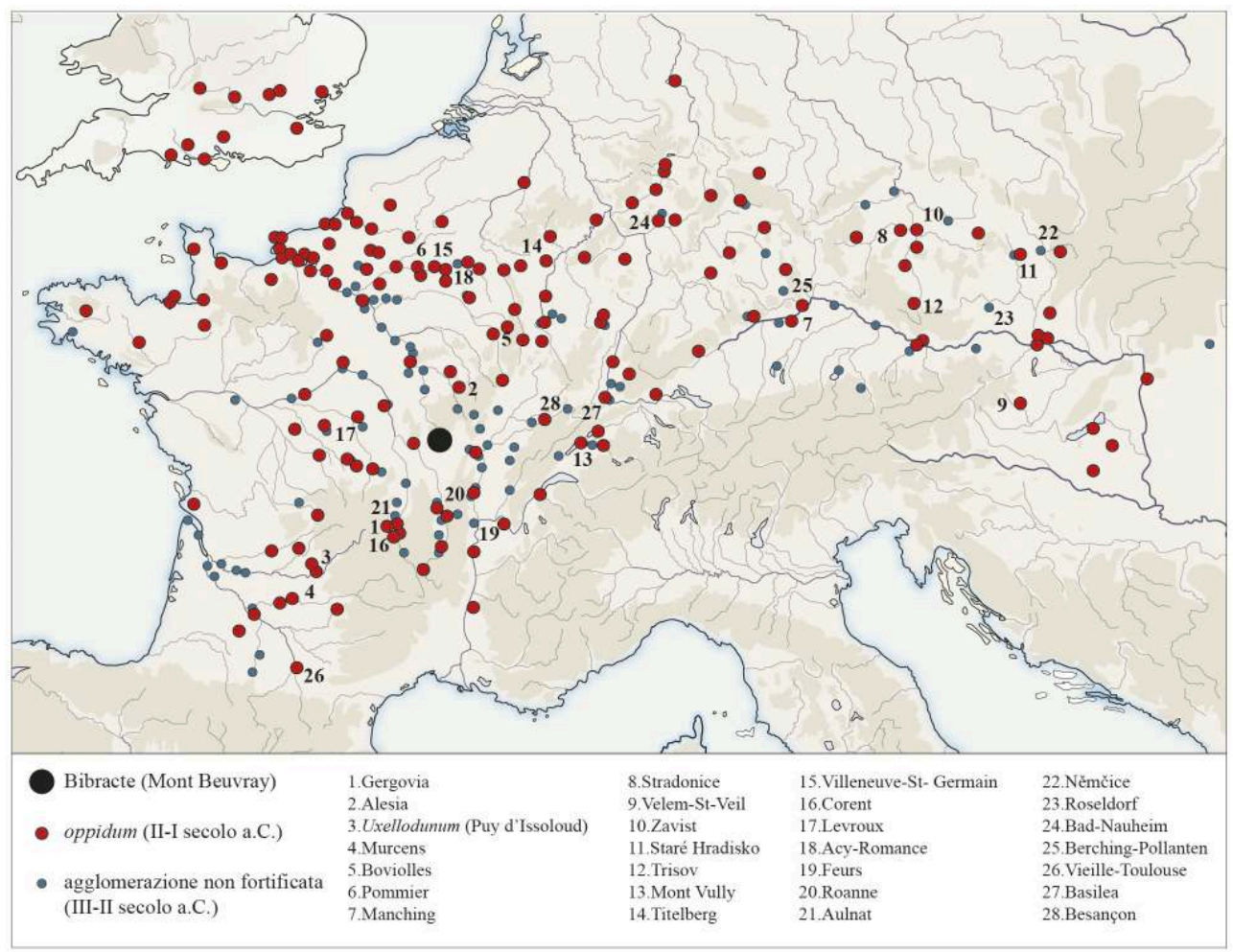

Fonti: <www.oppida.org>; Fichtl $(2013,21)$.

2 L'analisi storico-filologica dei Commentarii è all'origine dell'interesse antiquario che, nella Francia del XVIII secolo, sfocia nella pubblicazione dei primi rilievi topografici di fortificazioni celtiche da parte del marchese di Caylus $^{5}$ e delle prime carte della Gallia indipendente poste a corredo di opere monumentali quali l'Histoire romaine di Charles Rollin e Jean-Baptiste-Louis Crevier, pubblicata tra il 1731 ed il 1747. Alla metà del diciannovesimo secolo, con la pubblicazione dei primi manuali di archeologia nazionale ad opera di Louis Batissier (1843) o Jules Corblet (1851), l'oppidum celtico è ancora considerato essenzialmente nella sua accezione militare piuttosto che urbana. Corblet in particolare sottolinea l'ambiguità della definizione cesariana, ritenendo che il termine d'oppidum sia da riferirsi sia a centri d'abitato fortificati che a siti arroccati utilizzati esclusivamente in caso di conflitto ${ }^{6}$. L'autore propone allora la definizione - ereditata dalla tradizione antiquaria e oggi abbandonata - di oppida-murata per i primi e oppida-vallata per i secondi, generalmente più vasti in modo da accogliere una popolazione numerosa e vettovaglie abbondanti. Il vero punto di svolta si ha tuttavia negli anni'60 dell'Ottocento, quando l'imperatore Napoleone III, affascinato dalla figura storica di Cesare, alla quale dedicherà una biografia pubblicata nel 1866, finanzia una seria di campagne mirate al riconoscimento dei principali luoghi citati nei Commentarii. Tra il 1861 ed il 1865 il barone Eugène Stoffel, aiutante di campo dell'imperatore, coordina gli imponenti scavi di Gergovia (Puy-de-Dôme) e Alesia (AliseSainte-Reine, Côte-d'Or) seguiti dalle esplorazioni dell'oppidum di Uxellodunum (Puy d'Issoloud, Lot) alle quali si aggiungono gli scavi di Murcens (Lot) effettuati da Edouard Castagné nel 1868, il quale riconosce per primo il murus gallicus caratteristico delle fortificazioni urbane celtiche ${ }^{7}$. 
3 In questo contesto culturale e politico di riscoperta delle antichità nazionali, nel 1867 hanno avvio in Borgogna gli scavi sistematici dell'oppidum di Bibracte, «oppido Haeduorum longe maximo et copiosissimo»", affidati a Jacques Gabriel Bulliot. Smentendo la tradizione antiquaria predominante che localizzava la ricca capitale degli Edui sul sito di Augustodunum/Autun, fondata in epoca augustea, Bulliot intraprende un'estensiva esplorazione del Mont Beuvray ${ }^{9}$ (Saona e Loira, Nièvre), grazie alla quale vengono messe in luce le imponenti fortificazioni dell'oppidum e la complessa organizzazione del suo abitato intra muros. In trent'anni di ricerche, Bulliot documenta più di un centinaio di strutture legate tanto alla tradizione continentale d'architettura lignea che ai modelli architettonici importati dal mondo mediterraneo, organizzate in quartieri caratterizzati da una specifica funzione artigianale, residenziale o pubblica ${ }^{10}$; ciò nonostante, l'archeologo non associa in alcun momento l'oppidum di Bibracte al concetto di città espresso negli stessi anni nell'opera fondatrice di Fustel de Coulanges, pubblicata nel 1864. Al contrario, in un volume apparso nel 1878, scritto in collaborazione con Jules Roidot ${ }^{11}$, Bulliot considera i paradigmi teorici de La Cité antique inapplicabili nel caso di Bibracte: le civitates galliche dell'epoca non avrebbero infatti una struttura sociale sufficientemente sviluppata da risultare nella creazione di uno spazio politico urbano simile a quello greco o romano. In questo senso, l'oppidum si configura ancora essenzialmente come un luogo di rifugio e un emporium ${ }^{12}$.

4 Agli inizi del ventesimo secolo, questa concezione cambia radicalmente a seguito della pubblicazione, nel 1908, del secondo tomo dell'Histoire de la Gaule di Camille Jullian, nella quale la comparsa degli oppida celtici alla fine del II secolo a.C. è al contrario descritta come il risultato della progressiva concentrazione degli attributi della civitas in un unico luogo, che diventa per conseguenza capitale e metropoli: «Les hommes de la Gaule s'acheminaient donc vers ce régime municipal qui a fait la beauté du monde antique, où une ville maîtresse résume et dirige l'histoire d'une nation, par la force de ses murailles et de ses habitudes, le charme de ses lignes, la gloire de ses dieux, l'amour de ses habitants» ${ }^{13}$. Lo stesso concetto è ripreso da Joseph Déchelette, il quale, oltre a continuare l'opera di Bulliot sul Mont Beuvray interrompendo infine gli scavi nel 1907, introduce per primo un approccio archeologico-comparativo su scala continentale ponendo le basi di quella che sarà più tardi definita la «civilisation des oppida». Nel suo Manuel d'archéologie préhistorique, celtique et gallo-romaine, pubblicato nel 1914, l'autore dimostra, attraverso l'accostamento della cultura materiale di oppida francesi (Mont Beuvray), tedeschi (Manching), boemi (Stradonice) ed infine ungheresi (Velem-St-Veil), l'esistenza di un substrato culturale comune diffuso dalle coste atlantiche al bacino dei Carpazi e caratterizzato, dal punto di vista insediativo, dalla rapida comparsa di vaste città fortificate sviluppatesi a seguito di una «municipalizzazione» tardiva delle civitates celtiche durante il periodo precedente la conquista romana: «Les forteresses gauloises n'étaient point de simples lieux de refuge [...] mais de véritables villes occupées par une population fixe [...] $»^{14}$. Dopo la pubblicazione del Manuel, il concetto di oppidum celtico tende così a sdoganarsi della sua origine letteraria per diventare piuttosto un «oggetto archeologico» a se stante. Negli anni '30, Paul Reinecke e Joachim Werner riaffermano la dimensione urbana (Städtewesen) degli oppida della Germania meridionale, testimoniata soprattutto dagli importanti scavi di Manching, iniziati nel 1938 e rilanciati in seguito nel 1955. Nel secondo dopoguerra sono soprattutto gli oppida boemi e moravi (Zavist, Staré Hradisko, Trisov, ecc.) a essere oggetto di estese esplorazioni che nel 1970 conducono all'organizzazione del fondamentale convegno internazionale Keltische Oppida di Liblice-Praga, pubblicato da Jan Filip. L'apporto di risultati sempre 
più abbondanti stimola l'elaborazione di numerosi lavori finalizzati a sistematizzare il panorama in continua evoluzione. Una nuova definizione archeologica del termine oppidum viene proposta da Wolfgang Dehn nel 1962 attraverso l'enumerazione dei criteri fondamentali per la classificazione dei siti: una superficie non inferiore ai 30 ettari, un posizionamento solitamente arroccato, una cinta muraria di preferenza continua, costruita con tecnica a intelaiatura lignea (tipo murus gallicus o Pfostenschlitzmauer soprattutto) e dotata di porte a tenaglia (Zangentore) ${ }^{15}$. Negli anni ' 70 e '80, la pertinenza di questi criteri è oggetto di dibattito ${ }^{16}$ : la superficie minima degli oppida tende a essere ribassata (oggi portata generalmente a 15 ettari) e la presenza di un abitato intra muros con caratteristiche urbane diventa un parametro essenziale, anche se non determinante ${ }^{17}$. L'associazione tra oppidum e città si riafferma tuttavia come una costante nelle fondamentali sintesi pubblicate da John Collis nel $1984^{18}$, da Olivier Buchsenschutz e Françoise Audouze nel $1989^{19}$ e, più recentemente, da Stephan Fichtl nel $2000^{20}$. Gli ultimi decenni del ventesimo secolo vedono ugualmente un rinnovarsi delle ricerche grazie agli scavi di siti maggiori quali il Titelberg, in Lussemburgo, Villeneuve-Saint-Germain (Aisne) o più recentemente Corent (Puy-deDôme), ma soprattutto attraverso la creazione, a partire dal 1984 e con il patrocino del Presidente François Mitterrand, del programma di ricerca europeo che, settantasette anni dopo Déchelette, riattiva gli scavi sul Mont Beuvray tramite l'istituzione del museo e del Centre Archéologique Européen che ogni anno coordina il lavoro delle numerose équipes universitarie implicate nello studio dell'oppidum di Bibracte.

Gli anni 2000 segnano infine un ulteriore punto di svolta. Grazie al moltiplicarsi su scala nazionale e internazionale delle operazioni di archeologia preventiva, delle campagne di prospezione geofisica e dei programmi di ricerca universitari, la quantità di dati relativi al popolamento della seconda età del ferro nell'Europa continentale subisce un considerevole aumento ${ }^{21}$ che incita a rianalizzare - 0 «destrutturare», secondo l'espressione di Gilbert Kaenel ${ }^{22}$ - il concetto stesso di «civilisation des oppida» così come definito in passato. L'inurbamento del II e I secolo a.C., finora considerato un fenomeno tardivo e in un certo senso estraneo alla cultura celtica dei secoli precedenti, tende quindi a essere oggi rivalutato in un contesto diacronico e spaziale di mutazioni complesse, di proto-urbanizzazione e «disurbanizzazione».

\section{Il concetto di civitas nel mondo celtico e l'origine degli oppida}

6 La rivalutazione del fenomeno degli oppida passa in primo luogo attraverso il riesame della loro origine, legata fino agli anni ' 90 del ventesimo secolo soprattutto a fattori esogeni. In opere di grande diffusione come il catalogo della mostra I Celti, tenutasi a Venezia nel 1991, o la sintesi di Venceslas Kruta Les Celtes, histoire et dictionnaire del 2000, la spinta urbana che caratterizza il mondo celtico della tarda età del ferro viene associata essenzialmente ai riflussi socio-culturali dovuti alle espansioni di IV e III secolo a.C. verso l'Italia settentrionale e centrale o l'Europa sud-orientale ed ai conseguenti nuovi stimoli provenienti dalle città ellenistiche del Mediterraneo. Secondo Otto Hermann Frey, la fondazione degli oppida transalpini sarebbe quindi l'opera di popoli «inseriti e cresciuti all'interno di una civiltà urbana che diede forma alle strutture sociali descritte in seguito da Cesare ${ }^{23}$. Kruta riconosce in particolare nella Gallia Cisalpina «le laboratoire où se crée le fondamental indispensable pour l'apparition 
des formations celtiques de type proto-urbain $\rangle^{24}$. Più precisamente, la disfatta dei Boi Cispadani e la migrazione di parte della popolazione oltre le Alpi e verso la valle del Danubio nel 189 a.C. ${ }^{25}$ avrebbero veicolato già agli inizi del II secolo a.C. la costituzione delle prime forme d'abitato urbano in Europa centrale ${ }^{26}$, progressivamente diffusesi nel corso del secolo attraverso la Germania meridionale per raggiungere, infine, la Gallia transalpina. Questa ricostruzione, oggi generalmente superata ${ }^{27}$, presuppone un nesso di casualità tra la comparsa degli oppida e la trasposizione di un sistema politico municipale di ascendenza greca o romana in seno alle civitates celtiche. Gli studi più recenti tendono tuttavia a sottolineare la sostanziale differenza del concetto di civitas applicato - in primis da Cesare - al mondo celtico e al mondo mediterraneo. Se in quest'ultimo il termine indica innanzitutto un polo centrale - non necessariamente urbano -, la sua popolazione, il territorio che ne dipende ed il diritto che lo regge (ovvero la polis, il municipium), nel mondo transalpino la civitas si riferisce a un raggruppamento demograficamente e geograficamente più ampio, associabile piuttosto al concetto greco di ethne $e^{28}$, la cui natura è essenzialmente giuridico-politica più che etnica o culturale ${ }^{29}$. Questa differenza è stata recentemente esemplificata da John Collis in una serie di articoli ${ }^{30}$ ripresi da Manuel Fernández-Götz nel suo lavoro di dottorato ${ }^{31}$ attraverso la contrapposizione tra i concetti di «città-stato» e «stato-tribale»" Sinteticamente, lo «stato-tribale» ha innanzitutto un territorio e una popolazione più importante della "città-stato»: qualche decina di migliaia di chilometri quadrati a fronte di una media di $200 \mathrm{~km}^{2}$ (raramente oltre $1000 \mathrm{~km}^{2}$ ) per una popolazione dell'ordine delle centinaia di migliaia d'individui a fronte di qualche migliaio. Nello «stato-tribale», l'etnonimo tende a prevalere sul toponimo urbano, come testimoniato dal fatto che, nelle successive civitates gallo-romane, il primo viene spesso associato al nome della città principale (Lutetia Parisorum, Avaricum Biturigum, Augusta Treverorum, ecc.); viceversa, nelle emissioni monetali in seno alla civitas, toponimo e etnonimo sono sostituiti, salvo rare eccezioni, da patronimici di singoli individui. Nello «stato-tribale», il legame tra un gruppo umano ed una data area geografica è meno stabile, come dimostrano la mobilità dei popoli e le migrazioni di intere civitates attestate nelle fonti scritte e dall'archeologia. Infine, il popolamento dello «stato-tribale» tende a essere multipolare, includendo, in un'occupazione del territorio che resta essenzialmente rurale, più poli di attrazione (politica, economica o religiosa) tra cui santuari, villaggi di pianura più o meno ampi e centri economico-produttivi a carattere urbano (agglomerazioni non fortificate o oppida). Non si assiste quindi mai, in questo contesto, ad una identificazione tra civitas ed oppidum, quand'anche esso funga - come nel caso di Bibracte - da capitale o polo centrale. Questo tipo di ordinamento, attestato sicuramente in epoca cesariana, fonda le sue radici nel secolo precedente: già verso la metà del II secolo a.C., qualche decennio prima della fondazione di Bibracte, la civitas degli Edui ha per esempio un'organizzazione interna tale da essere in misura di firmare un trattato militare con Roma $^{33}$. Questa configurazione sarà in seguito mantenuta durante il riassetto provinciale d'epoca augustea, attraverso la costituzione delle civitates gallo-romane che, a loro volta, forniranno la base dell'organizzazione delle diocesi medievali ${ }^{34}$. La comparsa degli oppida alla fine del II secolo a.C. non si riconnette quindi ad un riassetto tardivo della civitas in senso municipale, come ritenuto da Jullian o Déchelette, ma si inserisce piuttosto come tappa finale di un lungo processo autoctono di urbanizzazione in seno alla società celtica. 


\section{La ciclicità del fenomeno urbano nel mondo celtico transalpino}

7 L'idea secondo cui, prima della fondazione degli oppida, il processo di accentramento urbano sia estraneo alla società celtica è oggi definitivamente abbandonata grazie ad una realtà archeologica sempre più ricca ed articolata. In rottura con il precedente modello di un'urbanizzazione tardiva e sostanzialmente legata ad influssi esterni, Vladimir Salač descrive, in una serie di articoli recenti ${ }^{35}$, il carattere ciclico del fenomeno urbano nell'Europa continentale (fig. 2), un processo che trova le sue radici già alla fine della prima età del ferro (periodo tardo hallstattiano, VI-V secolo a.C.). Gli studi degli ultimi decenni mostrano come alcune delle cosiddette «residenze principesche» (Fürstensitze) che caratterizzano questo periodo - piccoli siti arroccati di qualche ettaro posti a controllo dei principali assi commerciali e caratterizzati soprattutto per l'imponente concentrazione d'importazioni mediterranee di prestigio $^{36}$ - siano in realtà contemporanee d'agglomerazioni più ampie a carattere proto-urbano, come nel caso di Bourges (Cher) o Lione (Rhône) ${ }^{37}$. La scoperta di una città bassa murata ai piedi della collina fortificata dello Heuneburg, nel BadenWürttemberg, porta la superficie dell'agglomerazione, il cui apice si colloca alla prima metà del VI secolo a.C., a un centinaio di ettari, per una popolazione stimata a qualche migliaio di persone, paragonabile ai contemporanei centri del bacino mediterraneo ${ }^{38}$.

Fig. 2. - Modellizzazione del processo di urbanizzazione nella seconda età del ferro europea così come definito da Vladimir Salač.

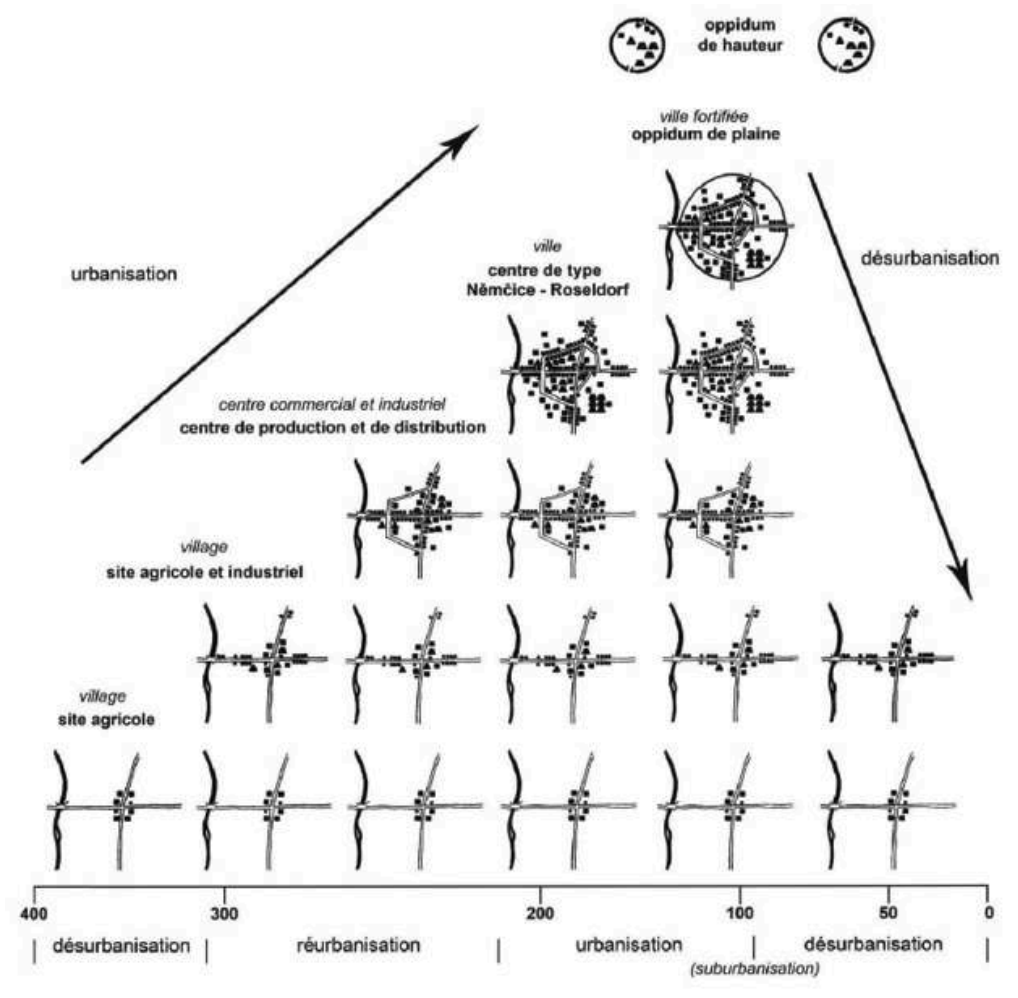

Salač $(2012,336)$.

A partire dal IV secolo a.C., alla distruzione ed al rapido abbandono degli Fürstensitze ${ }^{39}$ fa seguito un periodo di recessione, o «disurbanizzazione», che si prolunga fino agli 
inizi del III secolo a.C., momento durante il quale la tendenza si inverte. Quest'inversione, legata probabilmente ad un incremento demografico, produttivo e commerciale, si esprime, tra il III ed il II secolo a.C., nello sviluppo - o in alcuni casi nella fondazione ${ }^{40}$ - di agglomerazioni non fortificate (fig. 1) il cui carattere urbano, ancora oggetto di dibattito in attesa di futuri programmi di ricerca mirati, tende ad essere riconosciuto grazie alla presenza di spazi a vocazione pubblica o cultuale e di un abitato dall'assetto organizzato ${ }^{41}$. Nonostante in Francia l'attenzione verso queste agglomerazioni risalga agli anni '80 del ventesimo secolo, con gli scavi di siti quali Levroux (Indre), Acy-Romance (Ardennes), Feurs e Roanne (Loire) o Aulnat (Puy-deDôme), il loro ruolo chiave nel processo di urbanizzazione della seconda età del ferro è stato rivalutato soprattutto negli ultimi decenni. Une prima classificazione, basata sui recenti studi dei centri d'Europa centrale, è stata proposta da Vladimir Salač, il quale distingue tra «centri di produzione e distribuzione» (PDZ, Produktionu. Distributionszentren) e «centri tipo Němčice-Roseldorf» (NRZ, Němčice-Roseldorf Zentren), definizione quest'ultima fondata sui siti eponimi di Němčice, in Moravia, e Roseldorf, in Bassa Austria ${ }^{42}$. Se entrambe le categorie descrivono dei poli di redistribuzione economica (agricola e/o artigianale), i siti del secondo tipo - la cui massima fioritura si colloca alla seconda metà del II secolo a.C., cioè immediatamente prima dell'emergere degli oppida - si posizionano su una scala superiore in quanto a quantità e qualità della produzione artigianale, delle emissioni numismatiche e al raggio dei propri scambi commerciali. Queste agglomerazioni, in passato spesso considerate secondarie in un'antitesi villaggio non fortificato/città fortificata (oppidum), si caratterizzano al contrario come dei centri economici maggiori, la cui attività surclassa in molti casi quella attestata successivamente negli stessi oppida ${ }^{43}$. In Europa occidentale, esse si inseriscono in un'organizzazione territoriale alquanto eterogenea e multipolare, che comprende ugualmente grandi santuari ed un'occupazione delle campagne fortemente gerarchizzata, al cui apice si posizionano siti rurali di prestigio come Paule (Côtes-d'Armor) o Bâtilly-en-Gatinais (Loiret), espressione di una ricca élite aristocratica e paragonabili alle successive ville patrizie d'epoca romana.

9 La rapida - o piuttosto brutale - comparsa degli oppida alla fine del II secolo a.C., generalizzata all'intero mondo celtico transalpino, dalle coste atlantiche al bacino dei Carpazi, avviene quindi in un contesto insediativo ed economico ben organizzato e in pieno sviluppo; diversamente da quanto ritenuto fin in epoca recente, essa si configura piuttosto come una fase di rottura o di recessione in seno ad un processo di urbanizzazione già in atto da due secoli. Questa transizione si esprime in maniera eterogenea, ma nella maggior parte dei casi essa comporta l'abbandono dei siti non fortificati preesistenti ${ }^{44}$ e lo spostamento dell'abitato in posizioni più arroccate, siano esse a prossimità dei primi ${ }^{45}$ o fondazioni ex novo, come nel caso di Bibracte. Le ragioni storiche, politiche o sociali che sottintendono a questa brusca trasformazione del sistema di popolamento della tarda età del ferro devono essere ricercate, secondo Vladimir Salač, nella precisa volontà di affermazione politica in un contesto di rapidi cambiamenti di potere all'interno della civitas celtica, come l'assunzione da parte di una élite aristocratica del controllo della produzione e degli scambi: «La raison première de l'apparition des oppida, ce sont [...] les liens de pouvoir changeants, et par conséquent les besoins de l'élite ou de peuples entiers de manifester leur pouvoir $\rangle^{46}$. In questo senso, la costruzione delle imponenti fortificazioni che contraddistinguono gli oppida dalle agglomerazioni precedenti rivestirebbe un ruolo essenzialmente ostentatorio. 
Viceversa, in un articolo recente, Vincent Guichard ${ }^{47}$ sottolinea come la comparsa simultanea degli oppida nelle diverse regioni celtiche d'Europa non possa che essere interpretata alla luce di un contesto di crisi maggiore su scala continentale, da ricercare in una fase di grande mobilità delle popolazioni germaniche durante gli ultimi decenni del II secolo a.C. ${ }^{48}$. L'emergenza degli oppida rifletterebbe in questo senso una reazione di difesa, rappresentata da un processo generalizzato di incastellamento dell'abitato.

\section{L'origine e lo sviluppo (urbano) dell'oppidum di Bibracte}

Anche l'analisi di un sito come Bibracte (fig.3), che fin dai lavori fondamentali di Bulliot et Déchelette riveste un ruolo di primo piano nella definizione stessa del fenomeno degli oppida celtici, deve essere considerata oggi alla luce di questi nuovi modelli d'urbanismo transalpino: la fondazione dell'oppidum si colloca quindi in un periodo cerniera, la fine del II secolo a.C., caratterizzato da una rapida riorganizzazione del popolamento territoriale che, anche in area edua, si esprime attraverso l'abbandono quasi generalizzato di gran parte delle agglomerazioni preesistenti o il loro progressivo spostamento su posizioni più difese ${ }^{49}$, di cui Bibracte rappresenta sicuramente l'esempio più importante.

Fig. 3. - L'oppidum di Bibracte (Mont Beuvray), planimetria d'insieme.

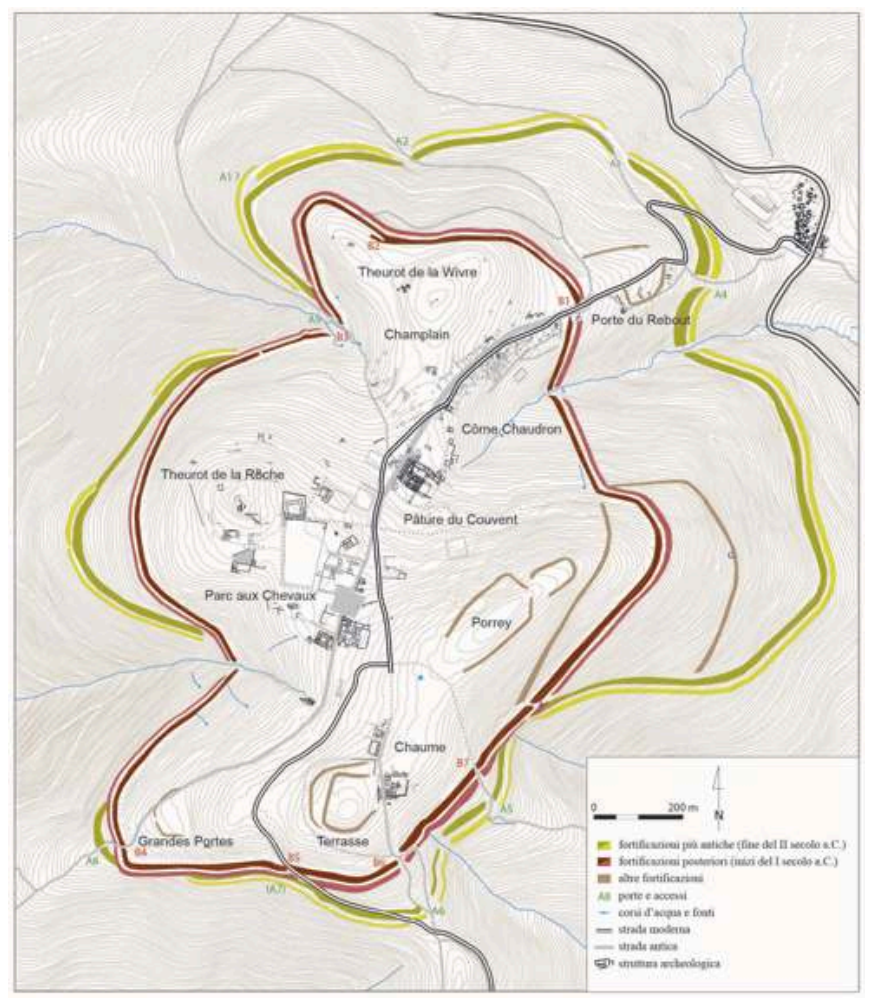

11 L'immagine ereditata dagli scavi ottocenteschi di un oppidum dal carattere fortemente urbano, contraddistinto da quartieri aristocratici popolati da una ricca élite romanizzata (il Parc aux Chevaux), quartieri densamente costruiti in cui si concentrano le attività artigianali (la Côme Chaudron o lo Champlain), ed infine spazi a vocazione 
essenzialmente pubblica (la Chaume, la Terrasse), cristallizza in realtà diverse fasi d'occupazione dell'oppidum che si susseguono all'indomani della conquista cesariana, periodo in cui Bibracte raggiunge il suo massimo sviluppo economico e demografico. I risultati degli scavi condotti dopo la ripresa delle ricerche nel $1984^{50}$ tendono viceversa ad evidenziare come la dimensione urbana, e per certi aspetti monumentale, dell'oppidum sia in realtà assente durante i primi decenni d'occupazione del sito. La fondazione di Bibracte alla fine del II secolo a.C. coincide essenzialmente con la costruzione di una cinta fortificata lunga $7 \mathrm{~km}$ (per una superficie intra muros di 200 ettari circa), edificata con mura ad armatura lignea (murus gallicus), concepite ed innalzate secondo un progetto unitario, il cui sforzo economico implica una volontà politica ben precisa. Il fatto che, alla costruzione delle fortificazioni, non faccia immediatamente seguito l'installazione di un abitato organizzato ${ }^{51}$ denota il carattere essenzialmente strategico della nuova fondazione.

All'inizio del I secolo a.C., questa configurazione iniziale subisce tuttavia un primo mutamento a seguito dell'abbandono della prima cinta muraria, sostituita da un nuovo perimetro fortificato più corto $(5,5 \mathrm{~km}$, edificato sempre con la tecnica del murus gallicus) che porta la superficie dell'oppidum a 135 ettari. In questa fase, le attestazioni d'attività in seno all'abitato tendono ad aumentare progressivamente, così come la quantità delle importazioni, preannunciando un grande riassetto in senso urbano del sito che si colloca nei decenni 70-60 a.C., periodo in cui altri centri, come per esempio il Titelberg o Besançon (Doubs), sembrano conoscere una simile riorganizzazione. L'urbanizzazione di Bibracte si esprime quindi in primo luogo attraverso la sistemazione degli spazi pubblici: l'arteria principale dell'oppidum viene ampliata fino a raggiungere una larghezza di $17 \mathrm{~m}$ e affiancata, in un quartiere centrale come la Pâture du Couvent, da portici in legno su cui affacciano boutiques o atelier artigianali. A questo momento si data la monumentalizzazione di una delle principali sorgenti del Mont Beuvray, la Fontaine Saint-Pierre, così come la costruzione, forse un po' più tarda, di un bacino pubblico in blocchi di granito, sempre alla Pâture du Couvent. Nello stesso periodo, la Porte du Rebout, accesso principale dell'oppidum, viene ugualmente riedificata: lo stretto passaggio che, nei primi decenni del secolo, garantiva la funzione difensiva delle fortificazioni, viene ampliato in un corridoio d'accesso largo $22 \mathrm{~m}$, coronato da due bastioni monumentali il cui carattere ostentatorio (paragonabile a quello delle successive grandi porte augustee di Augustodunum) sembra prevalere sull'originaria funzione militare. Il nuovo assetto urbano dell'oppidum è testimoniato inoltre dalla costruzione, sempre nei decenni 70-60 a.C., di un primo edificio a funzione pubblica, un quadriportico ligneo di dimensioni monumentali (una superficie di $1220 \mathrm{~m}^{2}$ ) messo in luce sul pianoro del Parc aux Chevaux a partire dal $2012^{52}$ (fig. 4). L'analisi architettonica degli edifici d'abitato costruiti in questo periodo permette inoltre di evidenziare il carattere pianificato della nuova organizzazione dell'oppidum. Essi derivano essenzialmente dalla tradizione continentale di architettura lignea e si caratterizzano per la tecnica di costruzione chiaramente normalizzata in una trama dei quartieri ben definita ${ }^{53}$. Gli edifici, che hanno una superficie al suolo generalmente ridotta di 20 a $30 \mathrm{~m}^{2}$, sono provvisti di ossature lignee massicce adatte a sostenere il peso di uno o più piani rialzati ${ }^{54}$ (fig. 5). Così come gli elementi costitutivi degli impalcati lignei, sistematicamente squadrati e dimensionati, le planimetrie rispecchiano dei moduli dimensionali prestabiliti nei quali è possibile riconoscere l'applicazione di un comune sistema di misura ${ }^{55}$. L'oppidum di Bibracte, fondato mezzo secolo prima essenzialmente con funzione strategica, si configura quindi come una vera 
e propria ville celtique - riprendendo l'espressione di Déchelette - soltanto nei decenni immediatamente precedenti alla conquista cesariana, assumendo probabilmente un ruolo preminente (di capitale) in seno alla civitas edua.

Fig. 4. - Il complesso pubblico del Parc aux Chevaux in corso di scavo nel 2017.

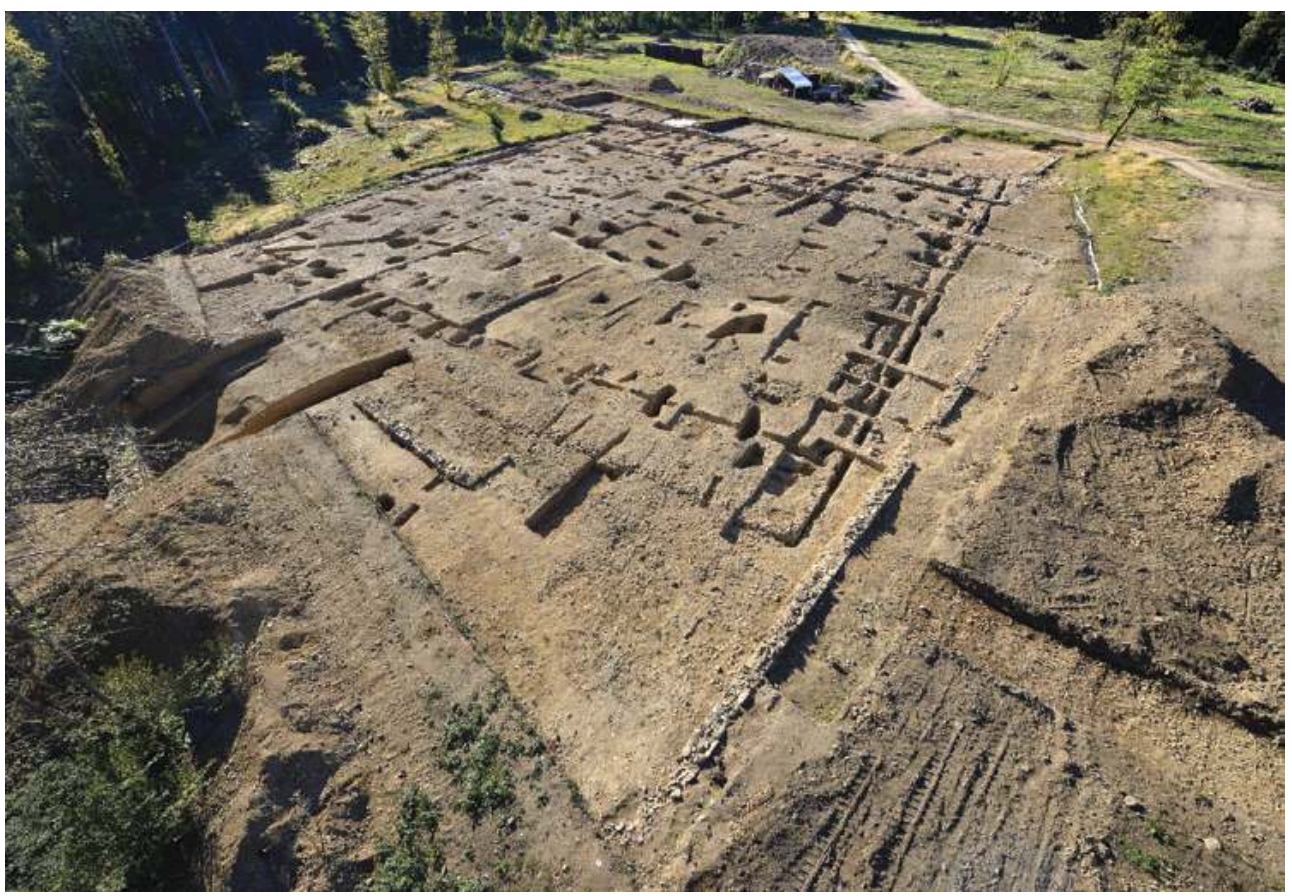

Resp. P. Barral, P. Nouvel, M. Thivet, M. Joly, Université de Franche-Comté e Université de Toulouse Immagine A. Maillier / Bibracte, n. 106995.

Fig. 5. - Esempio di struttura d'abitato di Bibracte in corso di scavo alla Come Chaudron.

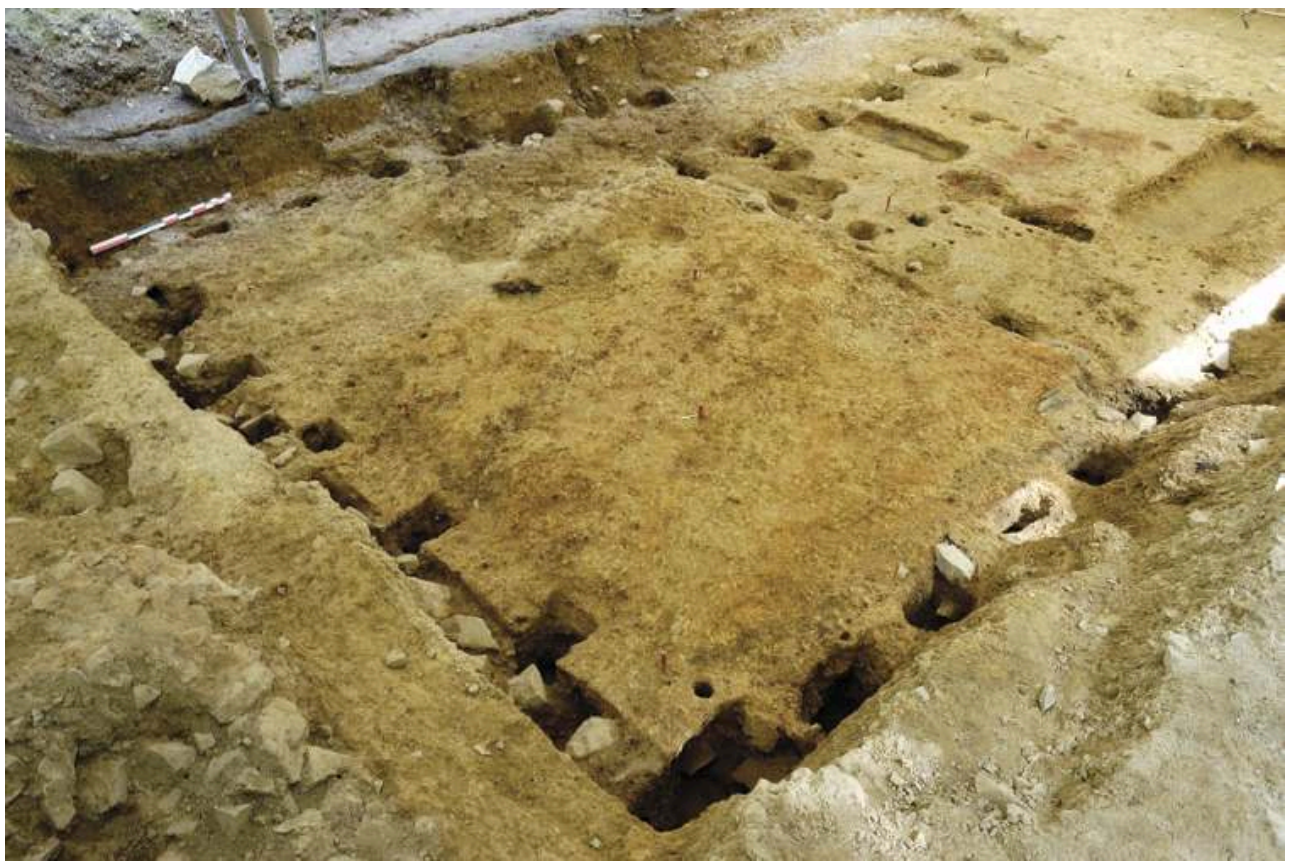

Resp. J. P. Guillaumet, P. Golàňovà, T. Bochnak, UMR 6298 ArTeHiS, Digione; Masarykova univerzita, Brno; Università di Rzeszów. Immagine A. Maillier / Bibracte, n. 63540. 
Durante la seconda metà del I secolo a.C., l'abitato si trasforma gradualmente a seguito dell'introduzione progressiva di nuovi modelli urbanistici e architettonici provenienti dal mondo italico. Un nuovo centro pubblico di fattura essenzialmente romana, composto da una basilica, spazi aperti e boutiques in una configurazione che ricorda quella del forum, viene costruito nel quartiere della Pâture du Couvent, senza tuttavia che ciò comporti l'abbandono del precedente complesso monumentale indigeno del Parc aux Chevaux, il quale, pur attraverso diversi rifacimenti, resterà attivo fino all'abbandono del sito. Allo stesso modo, la tradizione architettonica locale non viene immediatamente soppiantata dalle tecniche mediterranee, ma si sviluppa in forme d'abitato ibride: edifici in legno di $100 \mathrm{o} 120 \mathrm{~m}^{2}$, dotati di cantine, ma coperti già dagli anni 40 a.C. da tegole di tipo romano, o dimore a pianta centrale di modello italico, ma costruite ancora in ossature lignea e secondo il savoir-faire locale. La comparsa di un'architettura d'abitato di tipo pienamente romano, rappresentata dalle ricche domus occupate, secondo Déchelette, «par des riches Éduens fixés à Bibracte vers l'époque de César, au moment où commençait la pénétration de la civilisation italique dans la Gaule centrale» ${ }^{56}$, si colloca in realtà soltanto alla vigilia dell'abbandono dell'oppidum a profitto della nuova fondazione augustea di Augustodunum/Autun, in un momento in cui l'esperienza urbana di Bibracte può considerarsi già in via di conclusione.

\section{Nota conclusiva}

Il proposito di questa breve sintesi, inevitabilmente incompleta, è quello di delineare, attraverso l'esempio di Bibracte, la parabola della fondazione, dello sviluppo e infine dell'abbandono - ovvero dell'assorbimento nel nuovo mondo romano - degli oppida celtici, valutati tuttavia all'interno di un nuovo e complesso modello di urbanismo dell'Europa celtica nella seconda età del ferro i cui caratteri fondamentali hanno iniziato ad essere definiti soltanto in tempi recenti. Se la diffusione degli oppida rappresenta sicuramente un'esperienza urbana originale e propria al mondo celtico, essa costituisce soltanto la tappa finale di un lungo processo che gli scavi dei prossimi decenni permetteranno senza dubbio di precisare.

\section{BIBLIOGRAFIA}

BARRAL Philippe, HANTRAIS Juliette, JOLY Martine, NOUVEL Pierre \& THIVET Matthieu, Un nouveau type d'architecture publique? Le complexe monumental de PC15 à Bibracte et les bâtiments sur cour de la fin de l'âge du fer en Gaule interne, in A. Villard-Le Tiec, Y. Menez \& P. Maguer (edd.), Architectures de l'âge du fer en Europe occidentale et centrale (Actes du $40^{\circ}$ Colloque international de l'AFEAF, Rennes, 4-7 mai 2016), Rennes, Presses universitaires de Rennes, 2018, pp. 489-508.

BUCHSENSCHUTZ Olivier (ed.), L'Europe celtique à l'âge du fer (VIII ${ }^{e}-^{e r}$ siècles), Parigi, PUF, coll. « Nouvelle Clio », 2015. 
BUCHSENSCHUTZ Olivier, Les habitats de l'âge du fer en France du IV au Ir siècle avant J.-C., in J. Guilaine \& D. Garcia (edd.), La protohistoire de la France, Parigi, Hermann, 2019, pp. 403-419.

BUCHSENSCHUTZ Olivier \& AUDOUZE Françoise, Villes, villages et campagnes de l'Europe celtique : du début du II millénaire à la fin du $I^{e r}$ siècle avant J.-C., Parigi, Hachette, 1989.

BULLIOT Jacques Gabriel, Fouilles du Mont Beuvray (ancienne Bibracte) de 1867 à 1895, Autun, Imprimerie et librairie Dejussieu, 1899.

BULLIOT Jacques Gabriel \& ROIDOT Jules, La cité gauloise selon l'histoire et les traditions, Autun, Imprimerie et librairie Dejussieu, 1879.

COLLIS John, Oppida: Earliest Towns North of the Alps, Sheffield, University of Sheffield, 1984.

COLLIS John, Centralisation et urbanisation dans l'Europe tempérée à l'âge du fer, in S. Sievers \& M. Schönfelder (edd.), Die Frage des Protourbanisation in der Eisenzeit / La question de la protourbanisation à l'âge du fer, Bonn, Dr. Rudolf Habelt GmbH, 2012, pp. 1-14.

CORBLET Jules, Manuel élémentaire d'archéologie nationale, Parigi-Lione, Perisse Frères, 1851.

DÉCHELETTE Joseph, Manuel d'archéologie préhistorique, celtique et gallo-romaine, t. 2: Archéologie celtique ou protohistorique. Second âge du fer ou époque de La Tène, Parigi, Picard, 1914.

FERNÁNDEZ-GÖTZ Manuel, Identity and Power: The Transformation of Iron Age Societies in Northeast Gaul, Amsterdam, Amsterdam University Press, 2014a.

FERNÁNDEZ-GÖTZ Manuel, Understanding the Heuneburg: A Bibliographical Approach, in M. Fernández-Götz, H. Wendling \& K. Winger (edd.), Paths to Complexity: Centralisation and Urbanisation in Iron Age Europe, Oxford, Oxford Books, 2014b, pp. 24-34.

FERNÁNDEZ-GÖTZ Manuel, WENDLING Holger \& WINGER Katja (edd.), Paths to Complexity: Centralisation and Urbanisation in Iron Age Europe, Oxford, Oxford Books, 2014.

FICHTL Stephan, La ville celtique. Les oppida de 150 av. J.-C. à 15 apr. J.-C., Parigi, Errance, 2000.

FICHTL Stephan, L'émergence des civitates en Gaule et dans le monde celtique, in C. Haselgrove (ed.), Les mutations de la fin de l'âge du fer, Glux-en-Glenne, Centre archéologique européen du Mont Beuvray, 2006, pp. 41-54.

FICHTL Stephan, Du « refuge » à la ville : 150 ans d'archéologie des oppida celtiques, in L. Olivier (ed.), Le Musée d'Archéologie nationale et les Gaulois $d u \mathrm{XIX}^{e}$ au XXI ${ }^{e}$ siècle, Saint-Germain-en-Laye, Antiquités Nationales, 2012, pp. 81-98.

FICHTL Stephan, Les agglomérations gauloises de la fin de l'âge du fer en Europe celtique (III -I $^{e r}$ siècles av. J.-C.), in D. Garcia (ed.), L'habitat en Europe celtique et en Méditerranée préclassique, Arles, Errance, 2013, pp. 19-43.

FOCHESATO Andrea, L'économie de la construction en bois à Bibracte. Métrologie et standardisation, de l'architecture à la forêt, in J. Wilczek, A. Cannot, T. Le Cozanet, J. Remy, J. Machacek \& K. Klapste, Interdisciplinarité et nouvelles approches dans les recherches sur l'âge du fer, Brno, Masarykova univerzita, 2017, pp. 57-61.

FOCHESATO Andrea, Standardisation et organisation de la construction en bois à Bibracte Mont Beuvray (fin II el $^{e r}$ s. av. n. è.), in A. Villard-Le Tiec, Y. Menez \& P. Maguer (edd.), Architectures de l'âge du fer en Europe occidentale et centrale, Rennes, Presses universitaires de Rennes, 2018, pp. 469-488.

FREY Otto H., L'importanza dello stanziamento gallico in Val Padana per lo sviluppo culturale in Europa Centrale, «Scienze dell'Antichità. Storia, Archeologia, Antropologia», 2, 1988, pp. 519-534. 
GUICHARD Vincent, Les oppida, une parenthèse dans l'histoire de l'Europe tempérée ?, «Pallas», 105, 2017, pp. 159-171.

GUICHARD Vincent, SIEVERS Susanne \& URBAN Otto H. (edd.), Les processus d'urbanisation à l'âge $d u$ fer / Eisenzeitliche Urbanisationsprozesse, Glux-en-Glenne, Centre archéologique européen du Mont Beuvray, 2000.

GUICHARD Vincent, MEUNIER Arnaud \& PARIS Pascal, Chronique des recherches sur le Mont Beuvray 2013-2016, «Revue archéologique de l’Est», 67, 2018, pp. 151-211.

JULLIAN Camille, Histoire de la Gaule, t. 2: La Gaule indépendante, Parigi, Librairie Hachette, 1908.

KAENEL Gilbert, Agglomération et oppida de la fin de l'âge du fer : une vision synthétique, in C. Haselgrove (ed.), Les mutations de la fin de l'âge du fer, Glux-en-Glenne, Centre archéologique européen du Mont Beuvray, 2006, pp. 17-39.

KRUTA Venceslas, La Gaule intérieure, in G. Duby, Histoire de la France urbaine, t. I: La ville antique, Parigi, Seuil, 1980, pp. 195-229.

KYSELA Jan, Počátky českých oppida: Role Středomoří a urbanisační procesy v evropské době železné / Les origines des oppida en Bohême : le rôle de la Méditerranée et les processus d'urbanisation dans l'âge du fer européen, Tesi di dottorato, Universita Karlova v Praze, Université de Strasbourg, 2013.

LUKAS Dominik, A Historical-Semantic Approach to the Concept of 'Oppidum'. The Exemple of Bibracte, in M. Fernández-Götz, H. Wending \& K. Winger (edd.), Paths to Complexity: Centralisation and Urbanisation in Iron Age Europe, Oxford, Oxford Books, 2014, pp. 84-100.

MILCENT Pierre Yves, Résidence aristocratiques et expérience urbaine hallstattienne en France ( $\mathrm{VI}^{e}-\mathrm{V}^{e}$ siècle av. J.-C.), in S. Sievers \& M. Schönfelder (edd.), Die Frage des Protourbanisation in der Eisenzeit / La question de la proto-urbanisation à l'âge du fer, Bonn, Dr. Rudolf Habelt GmbH, 2012, pp. 91-113.

NOUVEL Pierre \& BARRAL Philippe, La dynamique d'urbanisation à la fin de l'âge du fer dans le centreest de la France, in S. Sievers \& M. Schönfelder (edd.), Die Frage des Protourbanisation in der Eisenzeit / La question de la proto-urbanisation à l'âge du fer, Bonn, Dr. Rudolf Habelt GmbH, 2012, pp. 139-164.

SALAČ Vladimir, Les oppida et les processus d'urbanisation en Europe centrale, in S. Sievers \& M. Schönfelder (edd.), Die Frage des Protourbanisation in der Eisenzeit / La question de la protourbanisation à l'âge du fer, Bonn, Dr. Rudolf Habelt GmbH, 2012, pp. 319-345.

SALAČ Vladimir, Oppida and Urbanisation Processes in Central Europe, in M. Fernández-Götz, H. Wending \& K. Winger (edd.), Paths to Complexity: Centralisation and Urbanisation in Iron Age Europe, Oxford, Oxford Books, 2014, pp. 64-75.

SIEVERS Susanne \& SCHONFELDER Martin (edd.), Die Frage des Protourbanisation in der Eisenzeit / La question de la proto-urbanisation à l'âge du fer, Bonn, Dr. Rudolf Habelt GmbH, 2012.

TARPIN Michel, Urbs et oppidum : le concept urbain dans l'antiquité romaine, in V. Guichard, S. Sievers \& O. H. Urban (edd.), Les processus d'urbanisation à l'âge du fer / Eisenzeitliche Urbanisationsprozesse, Glux-en-Glenne, Centre archéologique européen du Mont Beuvray, 2000, pp. 27-30.

VITALI Daniele, Celtes cisalpins, celtes transalpins : quelques réflexions sur le rôle de l'Italie du nord dans l'origine des oppida, in M. Groene (ed.), La préhistoire au quotidien : mélanges offerts à Pierre Bonenfant, Grenoble, Jérôme Millon, 1996, pp. 323-345. 


\section{NOTE}

1. Giulio Cesare, Commentarii de Bello Gallico, I, 5.

2. Il termine oppidum impiegato nel testo cesariano alternativamente ad urbs - attestato tuttavia più raramente - implica une precisa volontà di tradurre in termini giuridici una realtà estranea al mondo politico romano. In questo senso, come ben sottolineato da M. Tarpin (2000, 27-29), un oppidum può essere definito tale solo se riunisce dei requisiti fondamentali quali l'associazione di una comunità dotata di un'identità politica comune, appartenente quindi alla civitas, e la presenza di un limite sacro, giuridico e simbolico, ma non necessariamente concretizzato nella costruzione di mura urbane, come nel caso dei cosiddetti oppida immunita.

3. Giulio Cesare, Commentarii de Bello Gallico, I, 23: «cum exercitui frumentum metiri oporteret, et quod a Bibracte [...] non amplius milibus passuum XVIII aberat, rei frumentariae prospiciendum existimavit».

4. Giulio Cesare, Commentarii de Bello Gallico, VII, 63: «re in controversiam deducta totius Galliae concilium Bibracte indicitur».

5. Nel Recueil d'antiquités égyptiennes, étrusques, grecques et romaines pubblicato in sette volumi a Parigi tra il 1752 e il 1767.

6. «César donne indistinctement le nom d'oppida aux villes d'habitation et aux enceintes fortifiées, où les Gaulois ne se réfugient qu'en temps de guerre» (Corblet, 1851, 34).

7. Citiamo ugualmente gli scavi di Léon Maxe-Werly condotti sull'oppidum di Boviolles (Mosa) dal 1871 al 1877 e quelli effettuati da Octave Vauvillé a Pommiers (Aisne) nel 1886 e 1887: cfr. Fichtl (2012, 81-83).

8. Giulio Cesare, Commentarii de Bello Gallico, I, 23.

9. La collina del Mont Beuvray $(822 \mathrm{~m})$ si posiziona a una trentina di chilometri di distanza da Autun, nella regione storica del Morvan.

10. Bulliot (1899).

11. Bulliot \& Roidot (1878).

12. Lukas $(2014,92)$.

13. Jullian $(1908,63)$.

14. Déchelette (1914, 942, 947-948).

15. Fichtl (2000, 18-19).

16. Per una sintesi sulla questione: Kaenel (2006, 20-22).

17. Citiamo a titolo d'esempio l'oppidum del Mont Vully, in Svizzera (cantone di Friburgo), considerato tale malgrado l'assenza di vestigia d'abitato all'interno della cinta muraria: cfr. Kaenel $(2006,29)$.

18. Collis (1984).

19. Buchsenschutz \& Audouze (1989).

20. Fichtl (2000).

21. Si vedano per esempio le raccolte di articoli: Guichard, Sievers \& Urban (2000); Sievers \& Schönfelder (2012); Fernández-Götz, Wendling \& Winger (2014). Recentemente: Buchsenschutz (2015); Buchsenschutz (2019).

22. Kaenel $(2006,22)$.

23. Frey $(1988,531-533)$.

24. Kruta $(1980,199)$.

25. Tito Livio, Historiae, XXXVII, 57.

26. Secondo questo modello, la fondazione degli oppida boemi e moravi anticiperebbe quindi di quasi un secolo quella degli oppida francesi, ipotesi recentemente smentita da Jan Kysela (2013).

27. Si veda per esempio Vitali (1996).

28. Termine utilizzato da Strabone per descrivere le stesse realtà che Cesare definisce civitates.

29. Fichtl $(2006,43)$.

30. Per esempio Collis (2012). 
31. Fernandez-Götz (2014a, 56-63).

32. Quest'ultimo applicabile, oltre che al mondo celtico, anche ad altre aree culturali come la penisola iberica, l'Italia (Umbri, Sanniti, ecc.) o la stessa Grecia (Macedoni, Tessali, ecc.).

33. Tito Livio, Periochae, 61.

34. Fichtl $(2006,42)$.

35. Salač (2012); Salač (2014).

36. Per esempio il sito si Saint-Marcel sul Mont Lassois, a Vix (Côte-d'Or): Milcent (2012, 96-101).

37. Milcent (2012, 101-109).

38. Fernández-Götz (2014b).

39. Che segna il passaggio tra prima e seconda età del ferro in Europa.

40. Tito Livio riporta per esempio la fondazione (condere), nel 186 a.C., di un oppidum a 12 miglia dalla futura colonia di Aquileia da parte di Galli transalpini: cfr. Tito Livio, Historiae, XXXIX, 22, 6; Vitali (1996).

41. Fichtl (2013).

42. Il sito di Němčice, in Boemia, è conosciuto essenzialmente attraverso prospezioni superficiali; Roseldorf, in Bassa Austria, è fatto oggetto di scavi annuali a partire degli anni 2000: cfr. Salač $(2012,325-327)$.

43. Salač $(2012,328-329)$.

44. Più raramente si assiste alla fortificazione in situ di un'agglomerazione preesistente, come nel caso di Manching, in Baviera, o ad una continuità d'occupazione di centri non fortificati durante il I secolo a.C., come nel caso dei siti tedeschi di Bad-Nauheim o Berching-Pollanten; cfr. Salač $(2014,70)$.

45. Citiamo il caso degli oppida di Vieille-Toulouse (Alta Garonna), di Basilea (con il sito non fortificato di Gasfabrik e l'oppidum nel sito di Münsterhügel) o ancora dell'oppidum di Corent, sorto dopo l'abbandono del sito di Aulnat, nel bacino di Clermont-Ferrand (Puy-de-Dôme).

46. Salač $(2012,338)$.

47. Guichard (2017, 166-167).

48. Ricordata dalle fonti scritte nel periodo delle invasioni cimbriche.

49. Nouvel \& Barral (2012).

50. Per una sintesi recente: Guichard, Meunier \& Paris (2018).

51. Stando alle poche tracce precoci conservate, la superficie intra muros è interessata tra la fine del II secolo e gli inizi del I secolo a.C. essenzialmente da un abitato discontinuo (con zone di stoccaggio, tracce di attività artigianale ed edifici lignei di taglia generalmente ridotta) o da zone d'estrazione mineraria che sembrano tuttavia esaurirsi già durante i primi decenni dopo la fondazione del sito.

52. Barral et al. (2018).

53. Fochesato (2018).

54. Una configurazione che si adatta particolarmente ad un terreno in pendenza, come nei quartieri della Come Chaudron o dello Champlain: cfr. Fochesato (2018, 471-472).

55. Fochesato (2017).

56. Déchelette $(1914,953)$. 


\section{RIASSUNTI}

Fin dagli inizi del ventesimo secolo, il processo di urbanizzazione nell'Europa transalpina della seconda età del Ferro è stato associato alla comparsa tardiva, alla fine del II secolo a.C., dei cosiddetti oppida, centri fortificati il cui carattere urbano è stato riconosciuto grazie agli scavi di siti di riferimento come l'oppidum di Bibracte, nell'attuale Borgogna. L'esponenziale aumento delle ricerche archeologiche su scala europea degli ultimi decenni, tuttavia, incita a riesaminare la questione alla luce di un fenomeno urbano più articolato di quanto ritenuto finora, che trova le sue radici già nel III secolo a.C.

Depuis le début $\mathrm{du} \mathrm{xx}^{\mathrm{e}}$ siècle, le processus d'urbanisation dans l'Europe continentale du second âge du fer a été associé à l'apparition tardive, à la fin du II ${ }^{\mathrm{e}}$ siècle av. J.-C., des oppida, agglomérations fortifiées dont le caractère urbain a été reconnu grâce aux fouilles de sites de référence comme l'oppidum de Bibracte, en Bourgogne. L'augmentation exponentielle des données archéologiques à l'échelle européenne au cours des dernières décennies nous incite cependant à réexaminer la question compte tenu d'un phénomène urbain plus articulé, qui trouve ses racines déjà au $\mathrm{III}^{\mathrm{e}}$ siècle av. J.-C.

Since the beginning of the 20th century, the urbanization process during the second Iron Age in continental Europe has been associated with the late appearance, at the end of the 2nd century $\mathrm{BC}$, of the oppida, fortified towns whose urban character was recognised thanks to the excavations of some reference sites such as the oppidum of Bibracte, in Burgundy. However, the exponential increase in archaeological data on a European scale in recent decades prompts us to revisit the issue in the light of a more articulated urban phenomenon, which find its roots back in the $3 r d$ century BC.

\section{INDICE}

Mots-clés : urbanisation, âge du fer, oppida, Bibracte

Parole chiave : urbanizzazione, età del Ferro, oppida, Bibracte

Keywords : urbanization, Iron Age, oppida, Bibracte

\section{AUTORE}

\section{ANDREA FOCHESATO}

Archéologue, Bibracte EPCC

a.fochesato@bibracte.fr 